OPEN ACCESS

Edited by:

Lorenzo Cavagna,

Fondazione Ospedale San Matteo

(IRCCS), Italy

Reviewed by:

Fabrizio Luppi,

University of Milano Bicocca, Italy

Paola Rottoli,

University of Siena, Italy

Alain Meyer,

Hôpitaux Universitaires de

Strasbourg, France

*Correspondence:

Nadera J. Sweiss

nsweiss@uic.edu

these authors have contributed equally to this work and share first authorship

\footnotetext{
Specialty section

This article was submitted to

Rheumatology,

a section of the journal

Frontiers in Medicine
}

Received: 15 August 2020 Accepted: 22 January 2021

Published: 24 February 2021

Citation:

Vagts C, Ascoli C, Fraidenburg DR,

Baughman RP, Huang $Y$,

Edafetanure-Ibeh R, Ahmed S,

Levin B, Lu Y, Perkins DL, Finn PW

and Sweiss NJ (2021) Unsupervised

Clustering Reveals Sarcoidosis

Phenotypes Marked by a Reduction in

Lymphocytes Relate to Increased

Inflammatory Activity on

18FDG-PET/CT.

Front. Med. 8:595077.

doi: 10.3389/fmed.2021.595077

\section{Unsupervised Clustering Reveals Sarcoidosis Phenotypes Marked by a Reduction in Lymphocytes Relate to Increased Inflammatory Activity on 18FDG-PET/CT}

Christen Vagts ${ }^{1 \dagger}$, Christian Ascoli ${ }^{1 \dagger}$, Dustin R. Fraidenburg ${ }^{1}$, Robert P. Baughman ${ }^{2}$, Yue Huang ${ }^{1}$, Russell Edafetanure-Ibeh ${ }^{1}$, Samreen Ahmed ${ }^{3}$, Benjamin Levin ${ }^{4,5}$, Yang Lu ${ }^{6}$, David L. Perkins ${ }^{7}$, Patricia W. Finn ${ }^{1}$ and Nadera J. Sweiss ${ }^{1,3 *}$

${ }^{1}$ Division of Pulmonary, Critical Care, Sleep, and Allergy, Department of Medicine, University of Illinois at Chicago, Chicago, IL, United States, ${ }^{2}$ Department of Internal Medicine, University of Cincinnati Medical Center, Cincinnati, OH, United States, ${ }^{3}$ Division of Rheumatology, Department of Medicine, University of Illinois at Chicago, Chicago, IL, United States, ${ }^{4}$ Division of Cardiology, Department of Medicine, College of Medicine, University of Illinois at Chicago, Chicago, IL, United States, ${ }^{5}$ Jesse Brown VA Medical Center, Chicago, IL, United States, ${ }^{6}$ Division of Diagnostic Imaging, Department of Nuclear Medicine, The University of Texas MD Anderson Cancer Center, Houston, TX, United States, ${ }^{7}$ Division of Nephrology, Department of Medicine, University of Illinois at Chicago, Chicago, IL, United States

Introduction: Sarcoidosis is a T-helper cell mediated disease characterized by granulomatous inflammation. We posited that unsupervised clustering of various features in sarcoidosis would establish phenotypes associated with inflammatory activity measured by 18FDG-PET/CT. Our goal was to identify unique features capable of distinguishing clusters and subsequently examine the relationship with FDG avidity to substantiate their potential use as markers for sarcoidosis inflammation.

Methods: We performed a retrospective study of a diverse, but primarily African American, cohort of 58 subjects with biopsy proven sarcoidosis followed at the University of Illinois Bernie Mac Sarcoidosis Center and Center for Lung Health who underwent 18FDG-PET/CT scan. Demographic, therapeutic, radiographic, and laboratory data were utilized in unsupervised cluster analysis to identify sarcoidosis phenotypes. The association between clusters, their defining features, and quantitative measurements on 18FDG-PET/CT was determined. The relevance of these features as markers of 18FDG-PET/CT inflammatory activity was also investigated.

Results: Clustering determined three distinct phenotypes: (1) a predominantly African American cluster with chronic, quiescent disease, (2) a predominantly African American cluster with elevated conventional inflammatory markers, advanced pulmonary disease and extrathoracic involvement, and (3) a predominantly Caucasian cluster characterized by reduced lymphocyte counts and acute disease. In contrast to the chronic quiescent cluster, Clusters 2 and 3 were defined by significantly greater FDG avidity on 18FDG-PET/CT. Despite similarly increased inflammatory activity on 18FDG-PET/CT, Clusters 2, and 3 differed with regards to extrathoracic FDG avidity and circulating lymphocyte 
profiles, specifically CD4+ T-cells. Notably, absolute lymphocyte counts and CD4+ T-cell counts were found to predict 18FDG-PET/CT inflammatory activity by receiver operating curve analysis with a 69.2 and $73.42 \%$ area under the curve, respectively.

Conclusions: Utilizing cluster analysis, three distinct phenotypes of sarcoidosis were identified with significant variation in race, disease chronicity, and serologic markers of inflammation. These phenotypes displayed varying levels of circulating inflammatory cells. Additionally, reduction in lymphocytes, specifically CD4+ T-cells, was significantly related to activity on 18FDG-PET/CT. Though future studies are warranted, these findings suggest that peripheral lymphocyte counts may be considered a determinant of sarcoidosis phenotypes and an indicator of active inflammation on 18FDG-PET/CT.

Keywords: sarcoidosis, lymphopenia, 18FDG-PET/CT, immunopathogenesis, cluster analysis, phenotype

\section{INTRODUCTION}

Sarcoidosis is a heterogeneous multisystem disease characterized by granulomatous inflammation. While sarcoidosis affects a variety of races, African American women have the highest prevalence of disease and new cases most commonly are diagnosed in the 4 th and 5 th decades of life (1). Sarcoidosis has been characterized as a T-helper cell mediated disease (2). The ACCESS trial identified genetic risk factors for sarcoidosis development in various races, and some genes were linked to immune dysregulation and decreased lymphocytes in a Caucasian subset of this cohort $(3,4)$. Moreover, despite the lack of sarcoidosis specific thresholds, absolute lymphocyte counts $\leq 1.5 \mathrm{kcells} / \mu \mathrm{L}$ have been associated with disease activity and progression $(5,6)$. Peripheral depletion of $\mathrm{CD} 4+, \mathrm{CD} 8+$, and CD19+ T-cells has also been shown to be a characteristic of patients with severe sarcoidosis (7). Furthermore, lymphocyte gene expression is decreased in patients with severe disease and CD4+ T-cell exhaustion has been described as a manifestation of progressive disease $(8,9)$. Thus, further characterization of lymphopenia, as it relates to disease pathogenesis and inflammation, merits investigation within specific phenotypes of sarcoidosis.

Given the heterogeneous nature of sarcoidosis, establishing disease phenotypes is critical to identify common pathways that may further shed light on disease etiology and pathogenesis. Existing phenotypes in sarcoidosis have focused on subject and disease characteristics, to include radiographic staging, organ involvement, disease acuity, and need for treatment (1012). Even though useful clinical tools have been proposed, the classification criteria for various clinical phenotypes of sarcoidosis are not well-standardized and criteria for research purposes are lacking (13). Cluster analysis techniques have been utilized in diseases that are difficult to classify, including other autoimmune and pulmonary diseases, and have yielded novel insights (14-18). To this end, a recent report utilized cluster analysis of a large predominantly Caucasian sarcoidosis cohort and identified 6 subsets of subjects that better predicted disease progression than classical analysis (19). A second study utilized a supervised hierarchical cluster analysis to identify phenotypes of sarcoidosis based on 18FDG-PET/CT (PET scan) uptake.
Cluster and PET scan analyses provided a meticulous evaluation of organ involvement and a more precise evaluation of the extent of disease compared to traditional assessment (20). Cluster analysis has therefore proven helpful at identifying similarities of disease beyond conventional classification schemes which may have implications for treatment and prognostication.

PET scan is an emerging tool for assessing inflammation in sarcoidosis as well as other inflammatory and autoimmune disorders $(21,22)$. Uptake on PET scan, commonly defined as SUV $>2.5$, has been consistently associated with disease activity as well as pulmonary function in patients with sarcoidosis (23-25). The sensitivity of PET scan in identifying active inflammation ranges $89-100 \%$ and is overall higher when compared to traditional biomarkers (24). PET scan use has been evaluated in both acute and chronic disease, is superior at detecting ongoing inflammation in persistent disease, and shows promise in guiding treatment and prognosis (26-28). We posited the usefulness of PET scan as a tool in the absence of a biomarker specific enough to monitor inflammation in sarcoidosis (29). ATS guidelines support use of PET scan to assess extracardiac sarcoidosis, albeit with low quality of evidence, suggesting further research is needed to develop a disease specific biomarker with adequate sensitivity to serve as a more cost-effective assessment strategy (30).

Our goal was to identify unique phenotypes of sarcoidosis using an unsupervised cluster analysis in subjects with biopsy proven sarcoidosis in order to establish the relationship between these phenotypes and inflammatory activity, as measured by serologic markers of inflammation and avidity on 18FDGPET/CT scan. Our postulate was that a parsimonious set of demographic features along with clinically relevant therapeutic, radiographic, and laboratory features would identify subgroups of subjects with sarcoidosis at risk of active disease characterized by high levels of inflammation.

\section{METHODS}

\section{Subject Selection}

Study approval was obtained through the University of Illinois at Chicago (UIC) institutional review board. Adult subjects 18 years of age and older followed in the Bernie Mac Sarcoidosis 
Translational Advanced Research (STAR) Center at UIC with a history and tissue biopsy consistent with sarcoidosis, in accordance with ATS/ERS/WASOG criteria, were included (31). All subjects underwent a skull to thigh PET scan to evaluate clinically suspected metabolically active intrathoracic sarcoidosis, between December 2014 and June 2019. Subjects who had comorbid inflammatory disease that may result in increased metabolic activity on PET scan, including malignancy, connective tissue or autoimmune disease, or active infection were excluded. Subjects who did not have sufficient laboratory data available within 180 days prior or 30 days after the PET scan was completed were also excluded.

\section{Data Collection}

The electronic medical record was retrospectively reviewed to collect variables regarding subject demographics as well as clinically relevant therapeutic, radiographic, and laboratory data. In addition to sex and self-reported race, subject demographics included age, body mass index (BMI), and smoking status at time of PET scan. Time from diagnosis to PET scan was measured from the time of biopsy. Treatment at the time of PET scan was also abstracted from medical records and categorized into the following regimens (A) naïve or local treatment, (B) systemic corticosteroids, (C) non-steroidal immune modulator, (D) combination therapy with steroids, (E) combination therapy without steroids. Laboratory values obtained within 180 days prior to or 30 days after PET scan included complete blood counts, lymphocyte subsets, serologic markers of inflammation (ESR, CRP), and markers of extrathoracic organ involvement (complete metabolic panels and vitamin D levels). ACE levels at any point prior to the PET scan were also included (Supplementary Data Sheet 1 ).

\section{PET Scan Interpretation}

All 18FDG-PET/CT examinations at UIC were performed on a GE Discovery 690 FDG PET/CT scanner (GE Medical Systems, Milwaukee, WI). Dedicated PET scans from the skull base to the upper thighs were obtained 60-90 min after intravenous injection of $0.370-0.481 \mathrm{GBq}$ of FDG. Image acquisition was performed using non-cardiac-gated technique with PET parameters as follows: $2 \mathrm{~min} /$ bed for the non-cardiac fields and $10 \mathrm{~min} / \mathrm{bed}$ for fields covering the heart. Image acquisition was performed using non-cardiac-gated technique. CT scan was used for attenuation correction and parameters were as follows: $120 \mathrm{kV}, 120 \mathrm{mAs}$, pitch $0.813,16 \times 1.5-\mathrm{mm}$ collimation, slice thickness of $3 \mathrm{~mm}$ with an increment of $1.5 \mathrm{~mm}$.

Utilizing the LIFEx radiomics software, assessment of individual PET scans in DICOM format was performed to standardize and extract functional parameter measurements in order to quantitate the burden of 18FDG-avid areas per subject with the "total metabolic tumor volume (MTV) protocol" (32). First, the maximum standardized uptake volume (SUV ${ }_{\text {Max }}$ ) as well as the mean-SUV uptake in the liver were measured and together used to calculate the $S U V_{\text {Background-to- }} S_{U V} V_{M a x}$ ratio (SUV ratio). Liver mean-SUV uptake was calculated using a volume-of-interest of $3 \mathrm{~cm}^{3}$ from the subject's right hepatic lobe. SUV ratio, shown to be a reliable measure for prognostication and treatment response, and not $\mathrm{SUV}_{\mathrm{Max}}$, was utilized to minimize observer variability and standardize PET scan acquisition and reconstruction protocols (33-36). To maximize specificity of identifying a positive PET scan, lesions with a SUV ratio $\geq 2$ were considered positive for inflammatory activity related to sarcoidosis, whereas those with a SUV ratio $<2$ were regarded as negative. This approach is similar to the use of PET scan to assess chemotherapy response in various lymphomas (35). Total Metabolic Volume (TMV), a measure of the volume of increased FDG activity in milliliters, and Total Lesion Glycolysis (TLG), the product of mean-SUV uptake with the volume of uptake, were then measured as they may better reflect overall inflammatory activity and measurement partitioned into intra- and extrathoracic compartments. Lesions included in these calculations required a minimum SUV ratio of 2 , as previously noted, as well as and an SUV $>40 \%$ of the $\mathrm{SUV}_{\text {Max }}$. These thresholds were extrapolated for use in this study as they have been shown to improve accuracy of the metabolic tumor volume measurement in various malignancies (37-39). Activity captured by the LIFEx software was then corroborated with the original radiographic interpretation of the PET scan.

\section{Statistical Analysis}

All statistical analyses were performed with the R Statistical Environment (version 3.5.0) (40). A total of 22 subject phenotypic variables comprised of demographic, therapeutic, radiographic, and laboratory data abstracted from the electronic medical records were input as clustering features into the Modha-Spangler algorithm for mixed categorical (Table 1) and continuous (Figure 1) data in the kamila R-package to establish unsupervised sarcoidosis clusters. This algorithm seeks to effectively balance the contribution of continuous and categorical variables in an unsupervised fashion. In doing so, it adaptively selects the relative weight that simultaneously minimizes the within-cluster dispersion and maximizes the between-cluster dispersion for both the continuous and categorical variables (41). The Modha-Spangler framework was utilized to optimize the $\mathrm{k}$-medoids algorithm PAM (partitioning around the medoids) using Gower's distance with default parameters and an optimal weight of 0.8182 by a brute-force search strategy (42). Determination of the ideal number of clusters by average silhouette width was performed utilizing the pamk function with criterion specifying a "krange" of 3-6 clusters given cohort heterogeneity and sample size (43).

Clustering features utilized in the algorithm were tested for significant differences between clusters with $\chi^{2}$-test of independence for categorical data or via Kruskal-Wallis oneway analysis of variance for continuous data $(p \leq 0.05$ were considered statistically significant). Post-hoc analysis with Benjamini-Hochberg (BH) adjustment to account for the multiplicity problem that occurs with multiple comparisons was performed using Fisher's exact test for $\chi^{2}$-tests or Dunn's test for Kruskal-Wallis tests and an adjusted $p<0.1$ was pre-specified as significant. Correlations for continuous data were performed utilizing Spearman's rank correlation coefficient and considered significant if $p \leq 0.05$. To further investigate the probability of 
TABLE 1 | Baseline categorical parameters utilized as clustering features of the UIC-Sarcoidosis cohort $(n=58)$.

\begin{tabular}{|c|c|c|}
\hline \multicolumn{2}{|c|}{ Categorical parameters in clustering } & \multirow{2}{*}{$\begin{array}{c}\text { Frequency (\%) } \\
23(39.66)\end{array}$} \\
\hline Race & Caucasian & \\
\hline & African American & $35(60.34)$ \\
\hline \multirow[t]{2}{*}{ Sex } & Male & $21(36.21)$ \\
\hline & Female & $36(63.79)$ \\
\hline \multirow[t]{4}{*}{ Age } & 30-39 years & $12(20.69)$ \\
\hline & 40-49 years & 18 (31.03) \\
\hline & $50-59$ years & $22(37.93)$ \\
\hline & $60-69$ years & $6(10.35)$ \\
\hline \multirow[t]{5}{*}{ Body Mass Index } & Normal & $11(18.97)$ \\
\hline & Overweight & $10(17.24)$ \\
\hline & Class 1 Obesity & $11(18.97)$ \\
\hline & Class 2 Obesity & 16 (27.59) \\
\hline & Class 3 Obesity & $10(17.24)$ \\
\hline \multirow[t]{3}{*}{ Smoking history } & Never & $28(48.28)$ \\
\hline & Former & $24(41.38)$ \\
\hline & Current & $6(10.34)$ \\
\hline \multirow{4}{*}{$\begin{array}{l}\text { Time from diagnosis to } \\
\text { 18FDG-PET/CT }\end{array}$} & Quartile 1 & $17(29.31)$ \\
\hline & Quartile 2 & $14(24.14)$ \\
\hline & Quartile 3 & $13(22.41)$ \\
\hline & Quartile 4 & $14(24.14)$ \\
\hline \multirow{4}{*}{$\begin{array}{l}\text { Lung parenchyma on CT } \\
\text { Chest (at 18FDG-PET/CT) }\end{array}$} & Normal & $21(36.84)$ \\
\hline & Lung Nodules & $16(28.07)$ \\
\hline & Consolidation/GGO & $8(14.04)$ \\
\hline & Advanced & $12(21.05)$ \\
\hline \multirow[t]{5}{*}{ Treatment } & A & 15 (25.86) \\
\hline & B & 15 (25.86) \\
\hline & C & $6(12.07)$ \\
\hline & $D$ & $17(29.31)$ \\
\hline & $E$ & 4 (6.89) \\
\hline \multirow{2}{*}{$\begin{array}{l}\text { Angiotensin converting } \\
\text { enzyme level (Ever Elevated) }\end{array}$} & No & 38 (65.52) \\
\hline & Yes & 20 (34.49) \\
\hline
\end{tabular}

Prevalence of individual parameters is depicted as frequencies and percentages. 18FDG$P E T / C T$, positron emission tomography with 2-deoxy-2-fluorine-18-fluoro-D-glucose with integrated computed tomography; CT, computed tomography; GGO, ground glass opacities. Time from diagnosis to 18FDG-PET/CT ranged from 0 to 27 years and quartile distribution was as follows: $1=0-3$ years; $2=4-6$ years; $3=6-10$ years; $4 \geq 11$ years. Treatment regimens at time of 18FDG-PET/CT: $A=$ naive or local therapy; $B=$ systemic corticosteroid alone; $C=$ non-steroidal immune modulator alone; $D=$ combination therapy with corticosteroid; $E=$ combination therapy without corticosteroid.

PET-positivity based on cluster membership a logistic regression model was constructed and odds ratios calculated.

Receiver operator characteristic (ROC) analysis with 1,000 stratified bootstrap replicates and $95 \%$ confidence intervals was performed on the cohort using the $p R O C$ R-package to calculate the predictive accuracy of specific cell counts with regards to determining sarcoidosis inflammatory activity on PET scan. Threshold values for specific cell counts were determined utilizing the $p R O C$ package's coords function, and the optimal numerical threshold for each cell type was calculated by maximizing sensitivities and specificities as determined by Youden's J statistic (Youden's index) (44).

\section{RESULTS}

There were 58 subjects identified who had a PET scan and laboratory values within the time constraints. Biopsies were predominantly obtained from lung (44.8\%), lymph node (32.8\%), and liver (8.6\%) tissue. Thirty-five (60.34\%) subjects were African American and 36 (63.79\%) were women; however, the proportion of men and women within African American subjects was comparable to that of Caucasians in our cohort ( $\chi^{2}$-test $p=0.3503$ ). Most subjects were between 50 and 59 years of age $(37.93 \%)$, though $31.03 \%$ were between 40 and 49 years and $20.69 \%$ were between age 30 and 39 . There were no subjects under age 30 . Time from diagnosis to PET scan, ranging from 0 to 27 years, was utilized to assess disease chronicity and distributed into quartiles. In total, $29.31 \%$ of subjects were included in quartile 1 (0-3 years), $24.14 \%$ in quartile 2 (3-6 years), $22.41 \%$ in quartile 3 (7-10 years), and $24.14 \%$ in quartile 4 ( $\geq 11$ years). The most common treatment regimen was combination therapy with corticosteroid (regimen D; 17 of 58, 29.31\%), though $25.86 \%$ of subjects were on corticosteroids alone (regimen B) and another $25.86 \%$ of subjects were treatment naïve (regimen A). Though there was variability in treatment regimens between subjects, individual doses and medications remained stable within the study period for each subject. Other variables, to include BMI, smoking history, lung parenchyma characteristics, and treatment are outlined in Table 1. Laboratory values utilized in clustering are summarized in Figure 1. Absolute neutrophil values in the cohort were normal while the median absolute lymphocyte count was $1.5 \mathrm{kcells} / \mu \mathrm{L}$ (interquartile range from 1.0 to $2.1 \mathrm{kcells} / \mu \mathrm{L}$ ) which coincides with previously described lymphopenia defining thresholds in the general population and sarcoidosis (5, 6, $45,46)$. Absolute neutrophil counts were found to be affected by treatment regimen (KW-test $p=0.0255$ ); however, upon adjustment for multiple comparisons this difference was found to be specific for subjects on regimen $\mathrm{B}$ when compared to those on regimen A (median 5.5 vs. 3.1 kcells/ $\mu \mathrm{L}$, respectively; Dunn's test $p=0.0362$ ). In contrast, subjects who were treatment naïve had a comparable prevalence of lymphocyte reduction $(\leq 1.5 \mathrm{kcells} / \mu \mathrm{L})$ to those on treatment (53.33 vs. $53.49 \%$, respectively; $\chi^{2}$-test $p=0.9917)$. Multivariate regression analysis was performed to further assess the relationship between inflammatory cells and variables considered likely to alter their quantity (sex, race, age, BMI, smoking history, chronicity of disease, and treatment regimen) and did not identify significant associations $(p>$ 0.05). Otherwise, serologic markers of inflammation were mostly within the standard reference ranges with few outliers.

Using 22 independent variables as described in Table 1, Figure 1, 3 clusters were identified (Figure 2). Characteristics of each cluster are listed in Table 2. Significant features that defined clusters included race, disease acuity, treatment, lung parenchyma, various laboratory values reflecting extrathoracic 


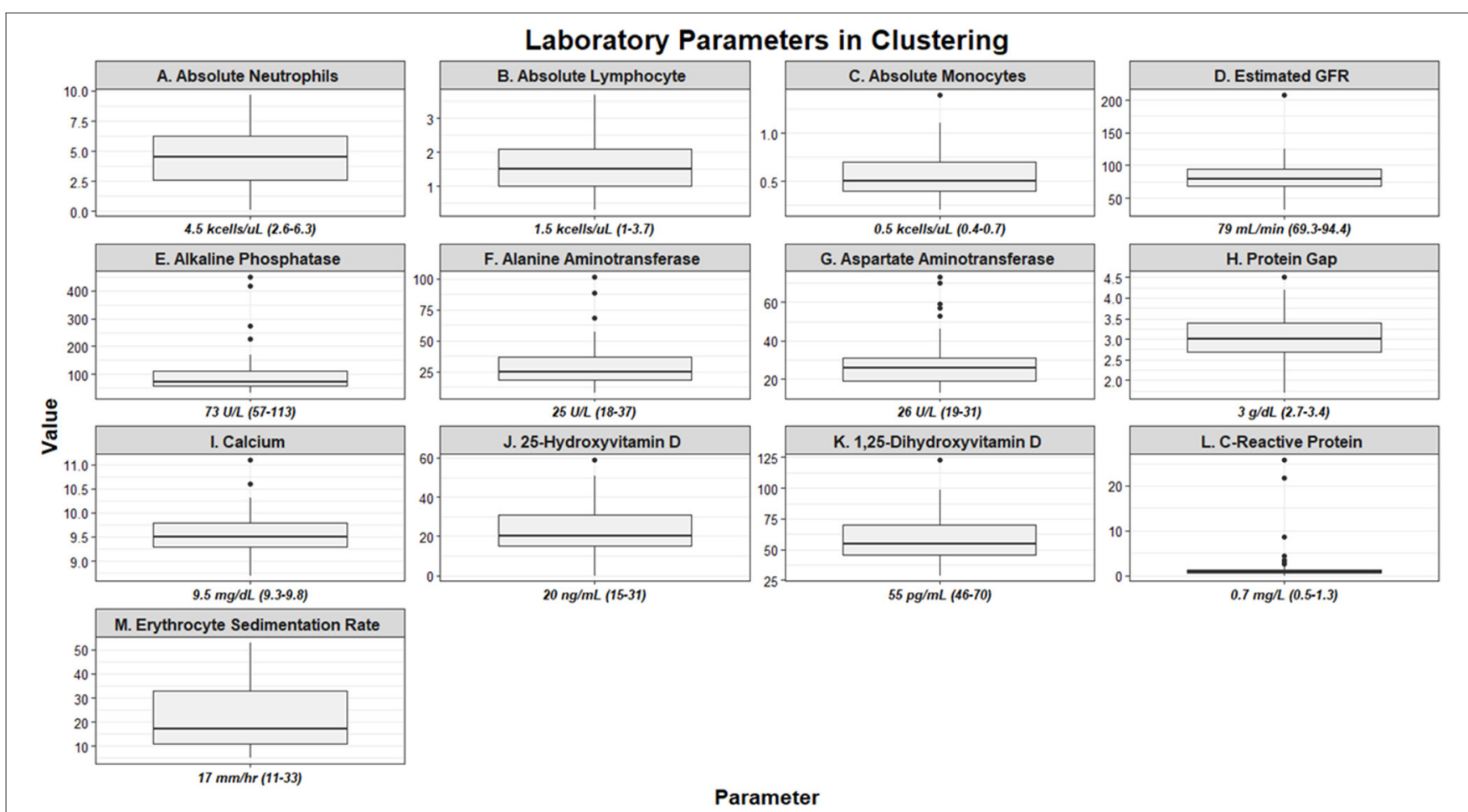

FIGURE 1 | Box and whisker plots demonstrating baseline laboratory parameters utilized as clustering features of the UIC-Sarcoidosis cohort $(n=58)$. Values are depicted as median and interquartile ranges for each individual parameter.

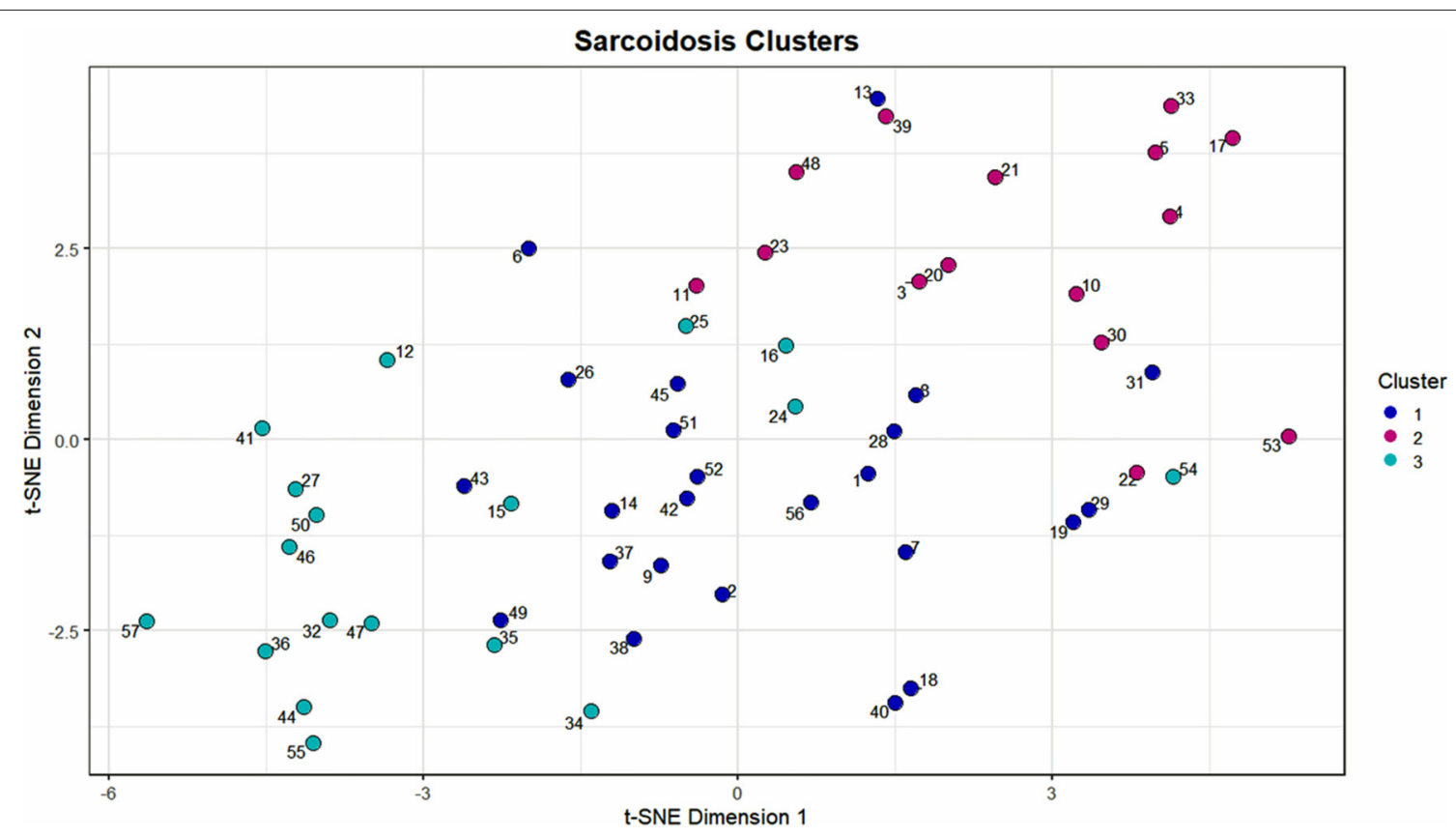

FIGURE 2 | Scatterplot visualization of the UIC-Sarcoidosis cohort over 2 dimensions utilizing the t-stochastic neighbor embedding (t-SNE) dimension reduction algorithm. The first and second dimensions are represented by the $x$-axis and $y$-axis, respectively. Points on the scatterplot represent individual subjects in the UIC-Sarcoidosis cohort and the distance between points is indicative of dissimilarity between subjects. Colors represent clusters identified by the Modha-Spangler algorithm utilizing partitioning around medoids with Gower's distance for base clustering of mixed data. Overall, Cluster 1 was identified as the largest cluster and comprised $41.38 \%$ of the cohort (24/58 subjects). Cluster 2 and 3 represented $25.86 \%(15 / 58)$ and $32.76 \%(19 / 58)$ of the cohort, respectively. 
TABLE 2 | Characteristics of UIC-Sarcoidosis cohort clusters.

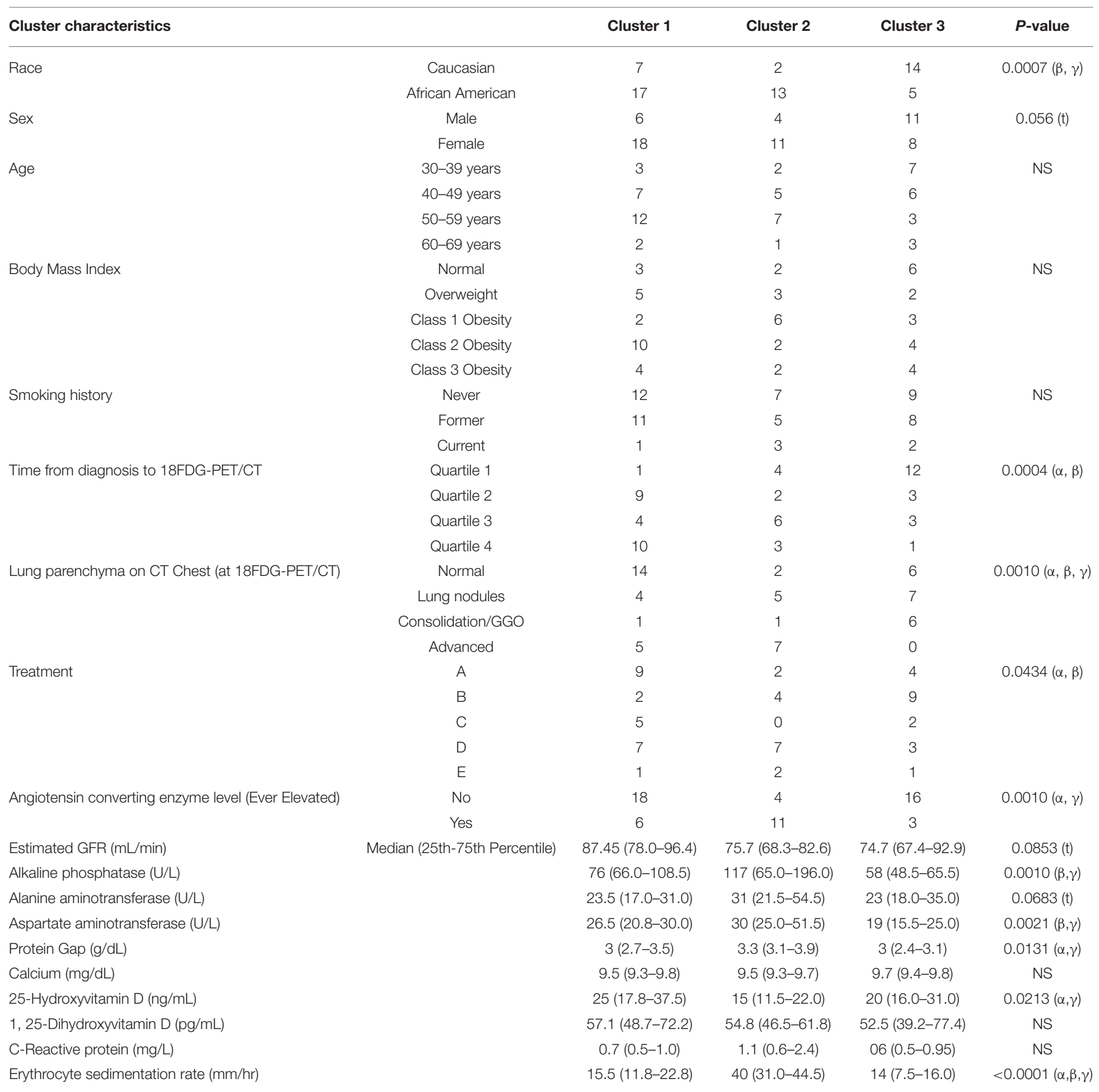

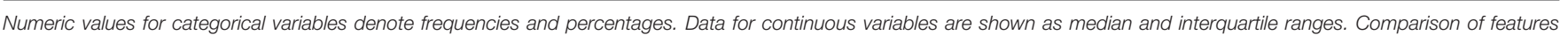

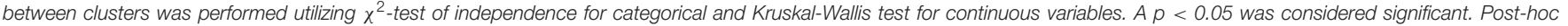

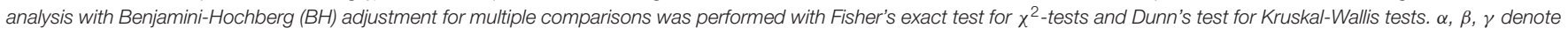

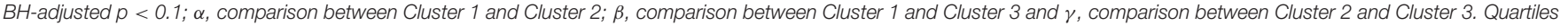
and treatment regimens as per Table 1. NS, non-significant; $t$, trend.

organ involvement, and serologic markers of inflammation. Cluster 1 and 2 consisted mostly of African American subjects (70.83 and $86.67 \%$, respectively) while only $26.32 \%$ of subjects in Cluster 3 were African American (KW-test $p=0.0007$ ). Subjects in Clusters 1 and 2 were mostly women (75.00 and $73.33 \%$, respectively) while most subjects in Cluster 3 were men (57.9\%); however, despite differences only a trend toward significance was noted (KW-test $p=0.0560)$. Cluster 1 had a considerably high number of subjects with PET scans performed $\geq 11$ years from diagnosis and less subjects with 
acute disease $(4.00 \%)$, while $78.94 \%$ of subjects in Cluster 3 were considered to have acute disease (KW-test $p=0.0004)$. Cluster 1 also consisted of more subjects with normal lung parenchyma on CT portion of the PET scan (77.78\%) while Cluster 2 had more advanced disease (46.67\%), reflected by moderate-severe emphysema and extensive fibrosis, and Cluster 3 had more lung nodules (36.85\%) and either consolidation or ground glass opacities (36.84\%). Cluster 1 included more treatment-naïve subjects whereas Cluster 3 had the most subjects on corticosteroid monotherapy (regimen B). Rate of any corticosteroid use (monotherapy or in combination) was highest in Cluster 2, and although a trend indicating possible difference in corticosteroid use was noted, statistical significance was not reached $\left(\chi^{2}\right.$-test $\left.p=0.0633\right)$. Cluster 2 also had more variation in laboratory values suggestive of extrathoracic organ involvement than Clusters 1 and 3 , as evidenced by more elevated alkaline phosphatase (median $117 \mathrm{U} / \mathrm{L}$, range 65.0196.0 U/L; KW-test $p=0.0010)$, aspartate aminotransferase (median $30 \mathrm{U} / \mathrm{L}$, range 25.0-51.5 U/L; KW-test $p=0.0021$ ), and protein gap (median $3.3 \mathrm{~g} / \mathrm{dL}$, range $3.1-3.9 \mathrm{~g} / \mathrm{dL}$; $\mathrm{KW}$ test $p=0.0131$ ). There was no significant difference in kidney function between clusters, though there was a trend toward lower GFR in Clusters 2 and 3 than Cluster $1(\mathrm{KW}$-test $p=$ 0.0853). Cluster 2 also had more subjects with abnormal serologic markers of inflammation, which included more subjects with a historically elevated ACE level (73.3\%; $\chi^{2}$-test $\left.p=0.0010\right)$ and a decreased 25-OH Vitamin D (median value $15.0 \mathrm{ng} / \mathrm{mL}$; KWtest $p=0.0213$ ). Variations in circulating inflammatory cells were evaluated across clusters with significant differences found in absolute neutrophil and absolute lymphocyte counts (Figure 3). In general, Cluster 1 had less abnormalities in inflammatory cell counts. Subjects in Cluster 3 had significantly higher absolute neutrophils than Cluster 1 but were not statistically different from Cluster 2 (KW-test $p=0.0181$, median values $5.5,3.8$, and $5.2 \mathrm{kcells} / \mu \mathrm{L}$, respectively). Subjects in Cluster 3 also had significantly lower absolute lymphocytes than Cluster 1 but were comparable to Cluster 2 (KW-test $p=0.0253$, median values $1.0,1.7$, and $1.4 \mathrm{kcells} / \mu \mathrm{L}$, respectively). In total, $68.42 \%(13 / 19)$ of subjects in Cluster 3 were found to have absolute lymphocyte counts $\leq 1.5 \mathrm{kcells} / \mu \mathrm{L}$ and among these $76.92 \%(10 / 13)$ had more evident reductions with counts $\leq 1.0$ kcells/ $\mu \mathrm{L}$. Whereas, in Cluster 2, a smaller proportion of subjects $(10 / 15)$ had absolute lymphocyte counts $\leq 1.5 \mathrm{kcells} / \mu \mathrm{L}$ of which only $30 \%(3 / 10)$ were found to have counts $\leq 1.0 \mathrm{kcells} / \mu \mathrm{L}$. Ultimately, comparison of clusters allowed the identification of 3 phenotypic clusters: (1) a more chronic, quiescent cluster, (2) an inflammatory extrathoracic cluster with advanced pulmonary disease, and (3) a cluster with acute disease and markedly reduced lymphocyte counts.

With the significant differences observed in absolute lymphocyte counts between clusters, associations between cluster and the CD4+ T-cell lymphocyte subset were further

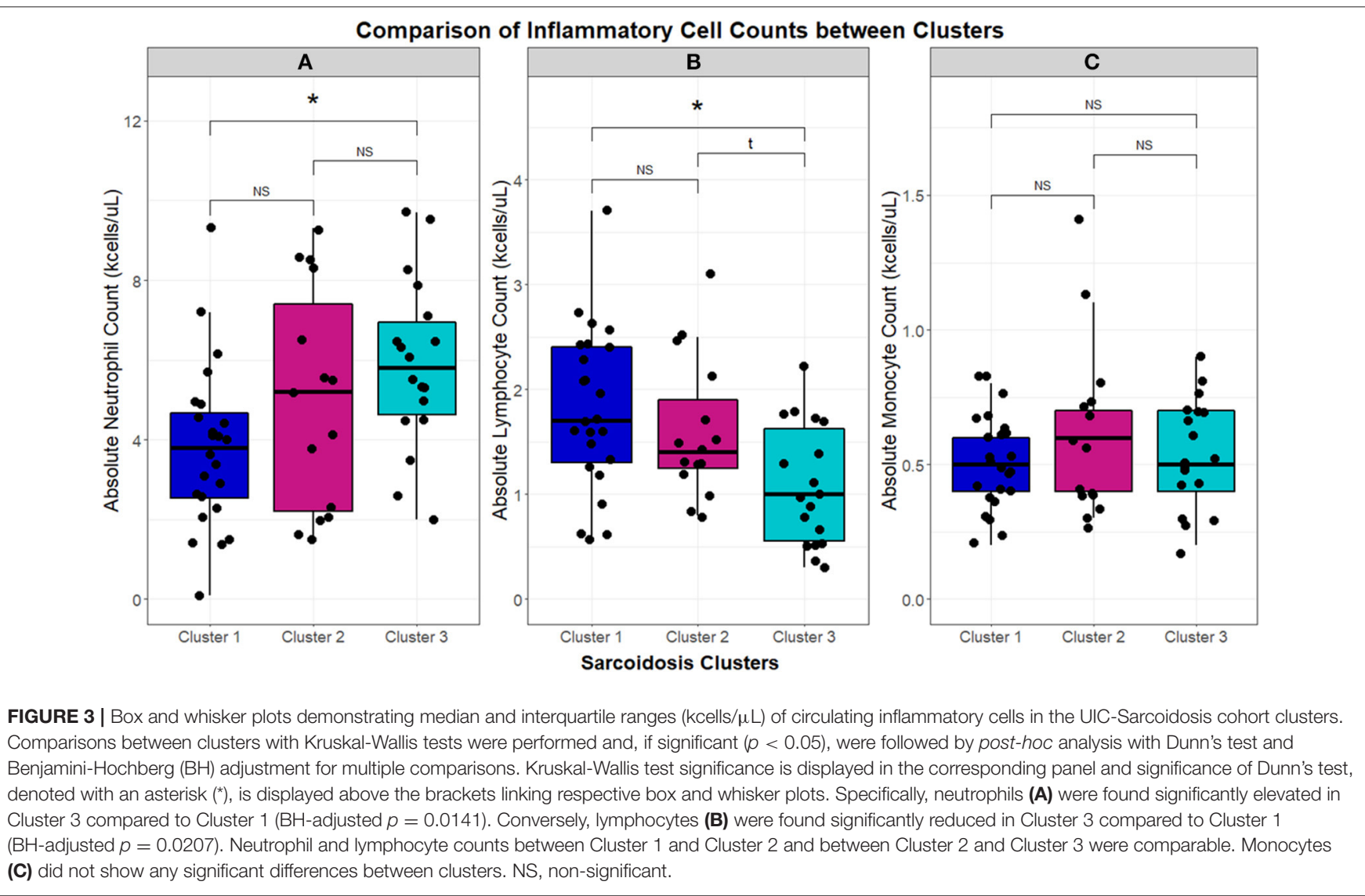


evaluated (Figure 4). CD4+ T-cells correlated with absolute lymphocytes across the UIC-Sarcoidosis cohort (Spearman's rho $=0.8329, p<0.0001)$. Between the clusters however, subjects in Cluster 3 had significantly lower CD4+ T-cells than subjects in both the chronic cluster (Cluster 1) and inflammatory extrathoracic-advanced cluster (Cluster 2), (KW-Test $p=$ 0.0008 , median values 421,818 , and 483 cells/ $\mu \mathrm{L}$, respectively). Interestingly, CD4+ T-cell counts in both Clusters 2 and 3 were found to be decreased in relation to reference ranges in the healthy population and sarcoidosis (7, 47, 48). CD4+ T-cells did not vary significantly between the chronic and advanced phenotypes.

With phenotypes supporting variable chronicity, disease location, and levels of inflammation, we proceeded to evaluate the association of these phenotypes with PET scan activity. Figure 5 describes the differences in SUV ratio between clusters. Notably, the inflammatory extrathoracic-advanced phenotype (Cluster 2) and acute-markedly reduced lymphocyte phenotype (Cluster 3) had significantly higher SUV ratios than Cluster 1 . With a median SUV ratio below 2, Cluster 1 was mostly comprised of subjects with negative PET scans. Conversely, Cluster 2 and 3 both had median SUV ratios $\geq 2$ suggesting more subjects had positive PET scans (odds ratio 6.00 and 6.50, respectively). SUV ratios did not vary between Clusters 2 and 3 .

As both the inflammatory extrathoracic-advanced phenotype (Cluster 2) and the acute-markedly reduced lymphocyte phenotype (Cluster 3) had high rates of PET avidity, we further investigated the relationship between inflammatory cells and PET positivity in the entire cohort. Receiver operator characteristic (ROC) analysis demonstrating the sensitivity and specificity of absolute neutrophils, absolute lymphocytes, and CD4+ T-cells as predictors of sarcoidosis inflammatory activity on PET scan are shown in Figure 6. Absolute lymphocytes and CD4+ Tcells predict PET positivity with an AUC of $69.20 \%$ and $73.42 \%$. There was no difference between their AUC $(p=0.36)$ and both represent strong associations with increased PET avidity and had significantly greater AUC than the absolute neutrophils ( $p$ $=0.0223$ for lymphocytes and $p=0.0029$ for CD4+ T-cells). Optimal cell count thresholds for the UIC-Sarcoidosis cohort, obtained with "Youden's index," suggest that absolute lymphocyte counts $\leq 1.25 \mathrm{kcells} / \mu \mathrm{L}$ and CD $4+$ T-cell counts $\leq 524.5 \mathrm{cells} / \mu \mathrm{L}$ are associated with PET scan positivity with median sensitivity of 51.72 and $68.97 \%$ and a median specificity of 82.76 and $72.41 \%$, respectively.

To further quantify the inflammatory activity on PET scans, we next assessed TMV and TLG, to compare FDG more stringently between phenotypes (Figure 7). The chronicquiescent phenotype (Cluster 1) had near normal TMV and TLG values suggesting minimal, if any, FDG avidity and were therefore essentially negative PET scans. This was consistent with the SUV ratio $<2$ and is therefore not surprising. These values were also significantly lower than the other groups. The inflammatory extrathoracic-advanced cluster (Cluster 2) and the acute-markedly reduced lymphocyte cluster (Cluster 3) had significantly higher TMV and TLG suggesting more sarcoidosis related inflammatory activity (KW-test $p=0.01397)$. PET avidity

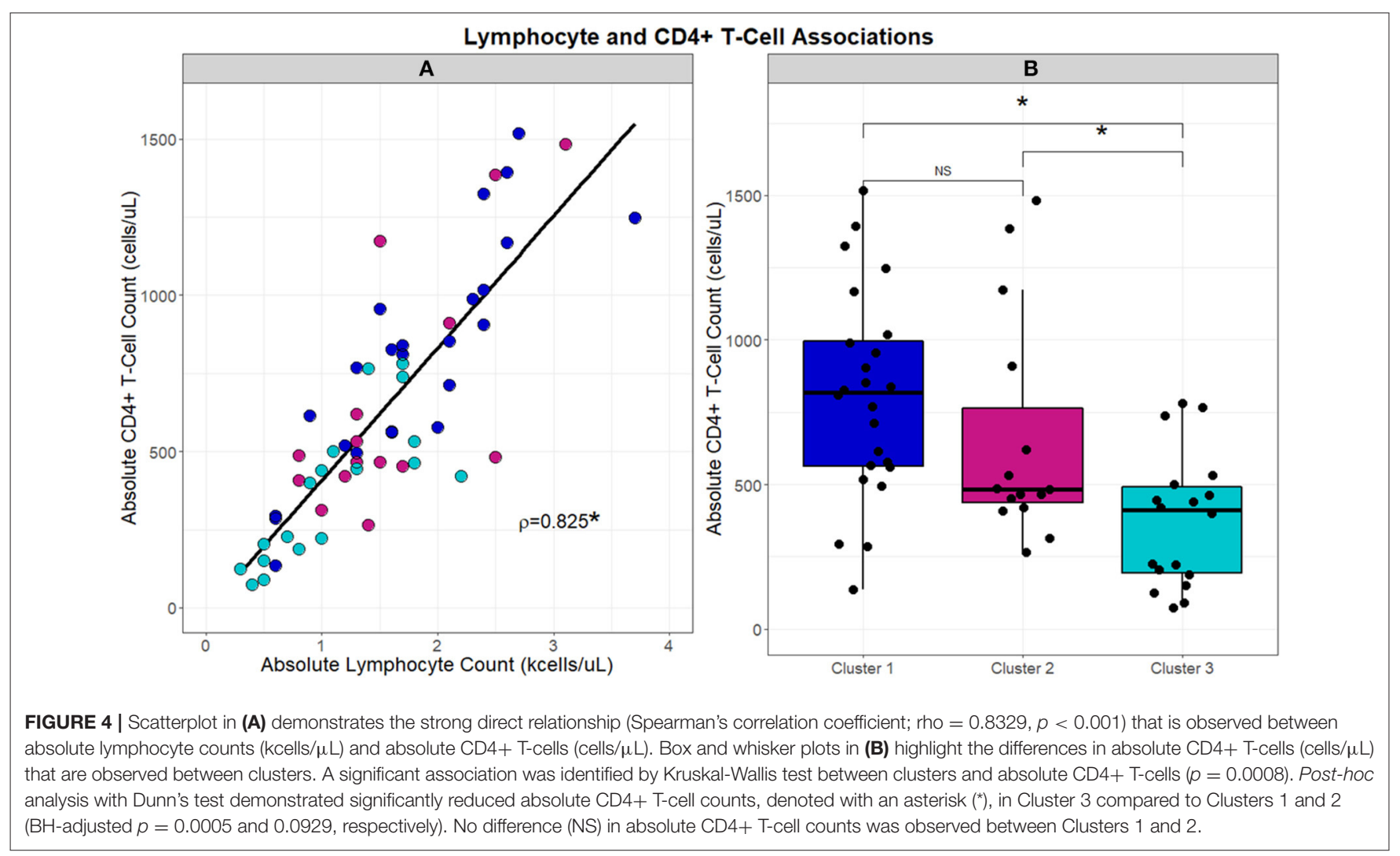




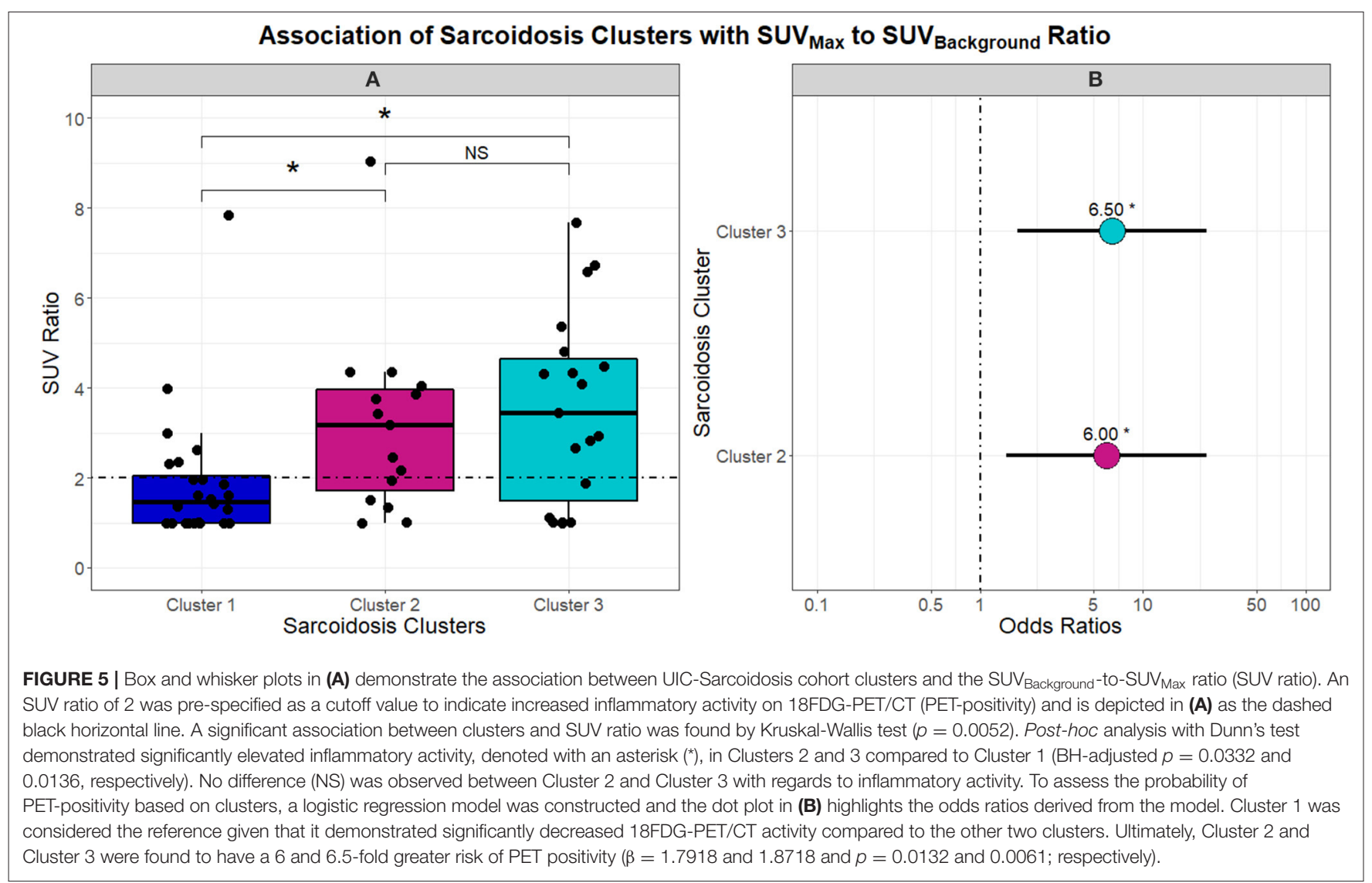

was further classified into intra and extra-thoracic activity to confirm true extrathoracic involvement in Cluster 2 as suggested by laboratory values. There was no significant difference between clusters in regards to intrathoracic metabolic volume (MV) and lesion glycolysis (LG) (Figures 7C,D, respectively); however, Cluster 2 had significantly higher extrathoracic MV (Figure 7E) and LG (Figure 7F) than both Cluster 1 and Cluster 3 (BH-adjp $=0.059$ and $\mathrm{BH}$-adj $p=0.055$, respectively). When comparing the phenotypes identified via cluster analysis with PET avidity, Cluster 1 is consistently quiescent while Cluster 2 and 3 are both hyperinflammatory; Cluster 2 is also confirmed to have more extrathoracic disease.

\section{DISCUSSION}

We have identified phenotypes of sarcoidosis in a diverse tertiary sarcoidosis referral center using unsupervised cluster analysis. We also examined the association between these phenotypes and the extent of sarcoidosis related inflammation. Our unbiased approach yielded 3 distinct clusters, (A) a predominant African American group of treatment naïve subjects with chronic sarcoidosis and minimal inflammatory activity on laboratory and PET scan values; (B) a predominant African American group of subjects with varying disease acuity requiring treatment, with advanced pulmonary parenchymal changes on CT and inflammation more extrathoracically located as evidenced by laboratory and PET scan values; and (C) a predominant Caucasian group of subjects with acute disease requiring treatment, with significant PET avidity that correlates with more significant absolute lymphocyte and CD4+ T-cell reduction. Dissimilarities observed between clusters (summarized in Table 3) attest to the variable immunogenicity and acuity of sarcoidosis. These findings underscore disparities in disease severity associated with race and to a degree, sex, that have previously been described (1). Prior phenotypic studies support the use of cluster analysis as an approach to identify clinical patterns and dissimilarities among large cohorts (16).

With significantly lower 18-FDG avidity, Cluster 1 identifies subjects with likely inactive sarcoidosis. Conversely, Clusters 2 and 3 are characterized by more subjects with positive PET scans and substantially greater TMV and TLG, consistent with more inflammatory activity and therefore active disease. Using the AUC of the ROC as an integrated measure of test performance, CD4+ T-cell count best predicted increased PET scan activity, though was not statistically superior to the absolute lymphocyte count threshold of $1.25 \mathrm{kcells} / \mu \mathrm{L}$ in our cohort. Notwithstanding, our independent analysis determined that a CD4+ T-cell count of $\leq 524.5$ cells $/ \mu \mathrm{L}$ is related to PET positivity and note that this was comparable to threshold levels previously utilized to assess major organ involvement in sarcoidosis (7). Despite the need for external validation, we conclude that absolute CD4+ 


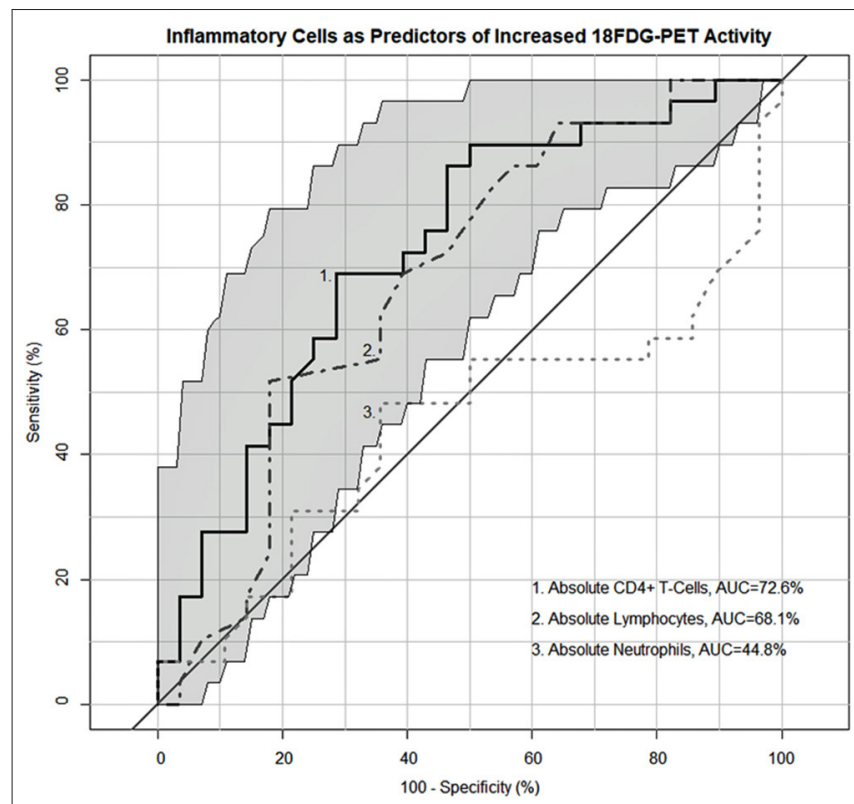

FIGURE 6 | Receiver operating characteristic (ROC) curves demonstrating the sensitivity and specificity of inflammatory cells as predictors of PET-positivity defined as an SUV ratio $>2$. Neutrophils (kcells $/ \mu \mathrm{L})$ demonstrated an area under the curve (AUC) of $45.78 \%$ whereas lymphocytes (kcells/ $\mu \mathrm{L}$ ) and CD4+ T-cells (cells/ $\mu \mathrm{L}$ ) demonstrated a 69.2 and $73.42 \%$ AUC, respectively. No difference was identified between the lymphocyte and CD4+ T-cell AUC utilizing the "bootstrap" method with 1,000 replicates $(p=0.36)$. Both lymphocytes and CD4+ T-cells demonstrated a significantly greater AUC compared to neutrophils ( $p=0.0223$ and 0.0029 , respectively). Optimal cell count thresholds obtained with "Youden's index" suggest that lymphocyte counts $\leq 1.25 \mathrm{kcells} / \mu \mathrm{L}$ in the UIC-Sarcoidosis cohort are most indicative of PET-positivity with a median sensitivity of $51.72 \%(95 \% \mathrm{Cl}$ : $34.48-68.97)$ and a median specificity of $82.76 \%$ (95\% Cl: 68.97-93.1). Similarly, CD4+ T-cell counts $\leq 524.5$ cells $/ \mu \mathrm{L}$ were most indicative of PET-positivity with a median sensitivity of $68.97 \%(95 \% \mathrm{Cl}: 51.72-82.76)$ and a median specificity of 72.41\% (95\% Cl: 55.17-89.66).

T-cell counts, or absolute lymphocyte counts in lieu of CD4+ T-cell enumeration, may serve as a predictor of sarcoidosis inflammatory activity.

Notably, in our cohort, while the quiescent phenotype was more treatment naïve, all phenotypes had similar rates of corticosteroid use, in combination with other immunomodulators or as monotherapy, and consequently were not considered to significantly influence the degree of lymphocyte reduction across clusters. As sarcoidosis is predominantly a T-helper cell mediated disease, mitigation of inflammation with use of corticosteroids, antimalarials, TNF- $\alpha$ antagonists, among others, has been a mainstay of treatment (49). Phenotype identification has the potential to guide therapy as has been previously shown in successful treatment of patients with sarcoidosis and CD4+ T-cell lymphopenia with the TNF- $\alpha$ antagonist, infliximab (50). Furthermore, use of PET scan has been described as an effective way to assess treatment response $(26,27)$. Thus, absolute lymphocyte counts and corresponding trends may be considered a useful parameter for monitoring therapy in addition to a surrogate for active inflammation in sarcoidosis.
A further comparison between Clusters 2 and 3 shows both clusters contain subjects with a reduction in the absolute lymphocyte count. However, the degree of reduction was only significant in the Caucasian/acute cluster and further characterized by a corresponding reduction in CD4+ T-cell counts. While we suspect immune dysregulation inherent to sarcoidosis drives peripheral lymphocyte depletion in this cluster, the effect seems to be present primarily in Caucasians, which is consistent with prior gene studies performed on the ACCESS cohort which was notably also predominantly Caucasian (4). Conversely, African Americans belonging to Cluster 3 did not have statistically lower absolute lymphocyte or CD4+ T-cell counts when compared to African Americans in other clusters. However, our cohort was comprised of only a small number of African Americans with acute disease and is a factor that limits full examination of this aspect and should be investigated in subsequent studies. Cluster 2 was characterized by more extrathoracic PET avidity, which is evident in the abnormalities in liver function tests and inflammatory markers. This group also had relatively more normal lymphocyte counts in comparison to Cluster 3, but overall still exhibited decreased absolute lymphocyte and CD4+ T-cell counts. As Cluster 2 was predominantly African American, there were too few Caucasians in this cluster to effectively assess features that distinguish Caucasians in this cluster from Caucasians in other clusters. Nonetheless, our findings suggest that race may influence immunotypes and underscores the importance of health disparities research in sarcoidosis.

Other than differences in lymphocytes, disease chronicity and radiographic findings on CT were also cluster defining features. Subjects in Cluster 1 had more chronic disease and more normal findings on CT, suggesting this cluster represents patients with resolved sarcoidosis. While Cluster 1 was predominantly African American, these findings were consistent when comparing Caucasians across clusters. Additionally, subjects in Cluster 2 had a higher frequency of advanced pulmonary parenchymal disease which may also be reflective of chronic active disease while subjects in Cluster 3 had more acute disease. Taken together these findings imply interdependence between disease chronicity and immunogenicity.

Despite our significant findings, there are several limitations to this study that should be the focus of future works. First, our study was limited by its retrospective design which did not allow full clinical assessment, such as specific organ involvement, at the time of PET scan and precluded the use of predictive tools such as the WASOG organ assessment instrument in the clustering algorithm which may have contributed to improved cluster determination and increased cluster uniformity (13). As with clinical data, acquisition of laboratory data relating to non-CD4+ T-cells was limited by the retrospective design and would have allowed better characterization of each proposed immunotype (7, 51-54). Additionally, although our cohort is representative of the predominantly African American population we serve and strengthened by strict inclusion criteria, sample size and heterogeneity limited our analysis. Consequently, determination of between cluster dissimilarities, particularly in relation to race and disease chronicity, was not possible. Notably, extrathoracic 


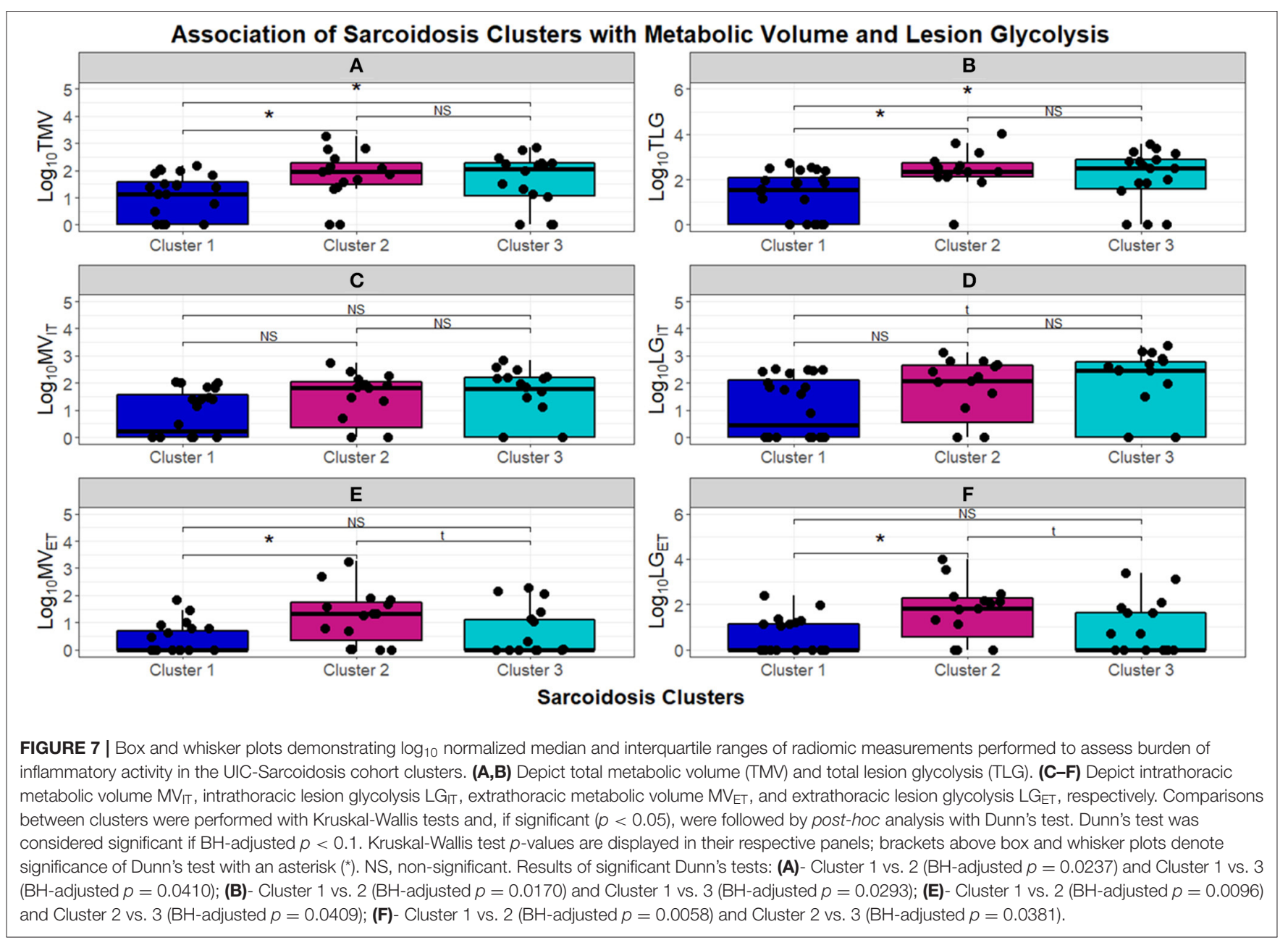

TABLE 3 | Summary of the most relevant characteristics that comprise the UIC-Sarcoidosis cohort clusters.

\begin{tabular}{|c|c|c|c|}
\hline Summary of cluster characteristics & Cluster 1 & Cluster 2 & Cluster 3 \\
\hline Race & African American & African American & Caucasian \\
\hline $\operatorname{Sex}^{(t)}$ & Female & Female & Male \\
\hline Disease Acuity & Chronic & Variable & Acute \\
\hline Lung Parenchyma & Normal & $\begin{array}{l}\text { Mixed } \\
\text { (Nodules \& Advanced) }\end{array}$ & $\begin{array}{l}\text { Mixed } \\
\text { (Nodules \& Consolidation/GGO) }\end{array}$ \\
\hline Treatment & Naïve & $\begin{array}{l}\text { Combination Corticosteroid \& } \\
\text { Immune modulator }\end{array}$ & Corticosteroid Monotherapy \\
\hline Markers of Inflammation & Normal & Elevated & Normal \\
\hline Absolute Lymphocytes (kcells/uL) ${ }^{(*)}$ & Normal & Reduced & Reduced \\
\hline Absolute CD4+ T-cells (cells/uL) ${ }^{(*)}$ & Normal & Reduced & Reduced \\
\hline 18FDG-PET/CT & Normal & Positive & Positive \\
\hline
\end{tabular}

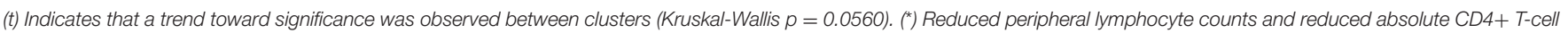
counts within the UIC-Sarcoidosis cohort clusters are defined as median values $\leq 1.5 \mathrm{kcells} / \mathrm{uL}$ and $\leq 500$ cells/uL, respectively.

disease assessment across the cohort was limited as FDG uptake on PET scan was considered excretional in the case of the kidneys or artifact in organs such as the heart and brain. Further limitations include lack of standardization of PET scan in terms of optimal protocols for sarcoidosis and lack of follow up PET scan due to radiation risk or insurance coverage.
In summary, we have identified a novel classification scheme using readily available demographic and clinically relevant data to identify three distinct sarcoidosis phenotypes with significant variation in race, disease chronicity, and inflammation. Whether the different immunotypes are reflective of disease acuity, race, comorbid conditions, or prior treatment remains to be resolved 
and follow-up studies should attempt to assess these differences in more homogeneous cohorts. However, peripheral reductions in lymphocytes, specifically CD4+ T-cells, were significantly related to inflammation identified on 18FDG-PET/CT and therefore sarcoidosis activity. While this finding is significant, a definitive threshold for clinically relevant lymphopenia has not been well-established in sarcoidosis. Though future prospective studies with larger cohorts are warranted, reductions in peripheral lymphocytes may be considered a determinant of sarcoidosis phenotypes and an indicator of active inflammation on 18FDG-PET/CT. Our study opens new doors for research that will help implement new classification criteria for the diagnosis and treatment of sarcoidosis. Multicenter validation studies will help to determine if this classification scheme can be applied broadly as well as clarify the clinical and immunologic implications of these findings.

\section{DATA AVAILABILITY STATEMENT}

The original contributions presented in the study are included in the article/Supplementary Material, further inquiries can be directed to the corresponding author/s.

\section{ETHICS STATEMENT}

The studies involving human participants were reviewed and approved by the University of Illinois at Chicago Office for the Protection of Research Subjects. Subjects provided written informed consent prior to participation in this study.

\section{REFERENCES}

1. Baughman RP, Field S, Costabel U, Crystal RG, Culver DA, Drent M, et al. Sarcoidosis in America. analysis based on health care use. Ann Am Thorac Soc. (2016) 13:1244-52. doi: 10.1513/AnnalsATS.201511-760OC

2. Grunewald J, Grutters JC, Arkema EV, Saketkoo LA, Moller DR, Muller-Quernheim J. Sarcoidosis. Nat Rev Dis Primers. (2019) 5:45. doi: 10.1038/s41572-019-0107-y

3. Baughman RP, Teirstein AS, Judson MA, Rossman MD, Yeager H Jr, Bresnitz EA, et al. Clinical characteristics of patients in a case control study of sarcoidosis. Am J Respir Crit Care Med. (2001) 164:18859. doi: 10.1164/ajrccm.164.10.2104046

4. Ascoli C, Huang Y, Schott C, Turturice BA, Metwally A, Perkins DL, et al. A circulating microRNA signature serves as a diagnostic and prognostic indicator in sarcoidosis. Am J Respir Cell Mol Biol. (2018) 58:4054. doi: 10.1165/rcmb.2017-0207OC

5. Morell F, Levy G, Orriols R, Ferrer J, De Gracia J, Sampol G. Delayed cutaneous hypersensitivity tests and lymphopenia as activity markers in sarcoidosis. Chest. (2002) 121:1239-44. doi: 10.1378/chest.121.4.1239

6. Selroos O, Koivunen E. Prognostic significance of lymphopenia in sarcoidosis. Acta Med Scand. (1979) 206:259-62. doi: 10.1111/j.0954-6820.1979.tb13507.x

7. Sweiss NJ, Salloum R, Gandhi S, Alegre ML, Sawaqed R, Badaracco M, et al. Significant CD4, CD8, and CD19 lymphopenia in peripheral blood of sarcoidosis patients correlates with severe disease manifestations. PLoS ONE. (2010) 5:e9088. doi: 10.1371/journal.pone.0009088

8. Hawkins C, Shaginurova G, Shelton DA, Herazo-Maya JD, Oswald-Richter KA, Rotsinger JE, et al. Local and systemic CD4(+) T Cell exhaustion reverses with clinical resolution of pulmonary sarcoidosis. J Immunol Res. (2017) 2017:3642832. doi: 10.1155/2017/3642832

9. Schott CA, Ascoli C, Huang Y, Perkins DL, Finn PW. Declining pulmonary function in interstitial lung disease linked to lymphocyte dysfunction. Am J Respir Crit Care Med. (2020) 201:610-3. doi: 10.1164/rccm.201910-1909LE

\section{AUTHOR CONTRIBUTIONS}

NJS, CA, CV, and DRF conceived and designed the study. Subject enrollment was performed by NJS, CA, DRF, RE-I, SA, and BL. Medical record abstraction was performed by CV, CA, RE-I, $\mathrm{SA}$, and $\mathrm{YH}$. Interpretation of imaging studies was performed by $\mathrm{CA}, \mathrm{CV}$, and YL. CA, CV, and DRF analyzed the data. CA, CV, RPB, DLP, PWF, and NJS wrote the manuscript. All authors contributed to the article and approved the submitted version.

\section{FUNDING}

Support for this study was provided by NIH grant numbers: R01 HL138628-01A1S1 and T32 HL144909; the Bernie Mac Foundation; and a donation by the University of Illinois at Chicago Vice-Chancellor of Health Affairs, Robert Barish, MD MBA.

\section{ACKNOWLEDGMENTS}

The authors would like to thank Martha Menchaca, MD Ph.D. for acquiring 18FDG-PET-CT images and transferring to DICOM format.

\section{SUPPLEMENTARY MATERIAL}

The Supplementary Material for this article can be found online at: https://www.frontiersin.org/articles/10.3389/fmed. 2021.595077/full\#supplementary-material

10. Baughman RP, Scholand MB, Rahaghi FF. Clinical phenotyping: role in treatment decisions in sarcoidosis. Eur Respir Rev. (2020) 29:190145. doi: 10.1183/16000617.0145-2019

11. Prasse A, Katic C, Germann M, Buchwald A, Zissel G, Muller-Quernheim J. Phenotyping sarcoidosis from a pulmonary perspective. Am J Respir Crit Care Med. (2008) 177:330-6. doi: 10.1164/rccm.200705-742OC

12. Schupp JC, Freitag-Wolf S, Bargagli E, Mihailovic-Vucinic V, Rottoli P, Grubanovic A, et al. Phenotypes of organ involvement in sarcoidosis. Eur Respir J. (2018) 51:1700991. doi: 10.1183/13993003.00991-2017

13. Judson MA, Costabel U, Drent M, Wells A, Maier L, Koth L, et al. The WASOG sarcoidosis organ assessment instrument: an update of a previous clinical tool. Sarcoidosis Vasc Diffuse Lung Dis. (2014) 31:19-27.

14. Adegunsoye A, Oldham JM, Chung JH, Montner SM, Lee C, Witt LJ, et al. Phenotypic clusters predict outcomes in a longitudinal interstitial lung disease cohort. Chest. (2018) 153:349-60. doi: 10.1016/j.chest.2017. 09.026

15. Guo Q, Lu X, Gao Y, Zhang J, Yan B, Su D, et al. Cluster analysis: a new approach for identification of underlying risk factors for coronary artery disease in essential hypertensive patients. Sci Rep. (2017) 7:43965. doi: $10.1038 /$ srep43965

16. Just J, Gouvis-Echraghi R, Rouve S, Wanin S, Moreau D, AnnesiMaesano I. Two novel, severe asthma phenotypes identified during childhood using a clustering approach. Eur Respir J. (2012) 40:5560. doi: 10.1183/09031936.00123411

17. Molano-Gonzalez N, Rojas M, Monsalve DM, Pacheco Y, Acosta-Ampudia Y, Rodriguez Y, et al. Cluster analysis of autoimmune rheumatic diseases based on autoantibodies. new insights for polyautoimmunity. J Autoimmun. (2019) 98:24-32. doi: 10.1016/j.jaut.2018.11.002

18. Kim S, Lim MN, Hong Y, Han SS, Lee SJ, Kim WJ. A cluster analysis of chronic obstructive pulmonary disease in dusty areas cohort identified three subgroups. BMC Pulm Med. (2017) 17:209. doi: 10.1186/s12890-0170553-9 
19. Rubio-Rivas M, Corbella X. Clinical phenotypes and prediction of chronicity in sarcoidosis using cluster analysis in a prospective cohort of 694 patients. Eur J Intern Med. (2020) 77:59-65. doi: 10.1016/j.ejim.2020.04.024

20. Papiris SA, Georgakopoulos A, Papaioannou AI, Pianou N, Kallergi M, Kelekis NL, et al. Emerging phenotypes of sarcoidosis based on 18F-FDG PET/CT: a hierarchical cluster analysis. Expert Rev Respir Med. (2020) 14:22938. doi: 10.1080/17476348.2020.1684902

21. Adams H, Keijsers RG, Korenromp IH, Grutters JC. FDG PET for gauging of sarcoid disease activity. Semin Respir Crit Care Med. (2014) 35:35261. doi: 10.1055/s-0034-1376866

22. Lu Y, Grant C, Xie K, Sweiss NJ. Suppression of Myocardial 18 F-FDG uptake through prolonged high-fat, high-protein, and very-low-carbohydrate diet before FDG-PET/CT for evaluation of patients with suspected cardiac sarcoidosis. Clin Nucl Med. (2017) 42:88-94. doi: 10.1097/RLU.0000000000001465

23. Keijsers RG, Verzijlbergen EJ, van den Bosch JM, Zanen P, van de Garde EM, Oyen WJ, et al. 18F-FDG PET as a predictor of pulmonary function in sarcoidosis. Sarcoidosis Vasc Diffuse Lung Dis. (2011) 28:123-9.

24. Keijsers RGM, Grutters JC. In which patients with sarcoidosis is FDG PET/CT indicated? J Clin Med. (2020) 9:890. doi: 10.3390/jcm9030890

25. Schimmelpennink MC, Vorselaars ADM, Veltkamp M, Keijsers RGM. Quantification of pulmonary disease activity in sarcoidosis measured with (18)F-FDG PET/CT: SUVmax versus total lung glycolysis. EJNMMI Res. (2019) 9:54. doi: 10.1186/s13550-019-0505-X

26. Chen H, Jin R, Wang Y, Li L, Li K, He Y. The utility of (18)FFDG PET/CT for monitoring response and predicting prognosis after glucocorticoids therapy for sarcoidosis. Biomed Res Int. (2018) 2018:1823710. doi: 10.1155/2018/1823710

27. Guleria R, Jyothidasan A, Madan K, Mohan A, Kumar R, Bhalla AS, et al. Utility of FDG-PET-CT scanning in assessing the extent of disease activity and response to treatment in sarcoidosis. Lung India. (2014) 31:32330. doi: 10.4103/0970-2113.142092

28. Mostard RL, Voo S, van Kroonenburgh MJ, Verschakelen JA, Wijnen PA, Nelemans PJ, et al. Inflammatory activity assessment by F18 FDGPET/CT in persistent symptomatic sarcoidosis. Respir Med. (2011) 105:191724. doi: 10.1016/j.rmed.2011.08.012

29. Chopra A, Kalkanis A, Judson MA. Biomarkers in sarcoidosis. Expert Rev Clin Immunol. (2016) 12:1191-208. doi: 10.1080/1744666X.2016.1196135

30. Crouser ED, Maier LA, Wilson KC, Bonham CA, Morgenthau AS, Patterson $\mathrm{KC}$, et al. Diagnosis and detection of sarcoidosis. an official american thoracic society clinical practice guideline. Am J Respir Crit Care Med. (2020) 201:e2651. doi: 10.1164/rccm.202002-0251ST

31. Hunninghake GW, Costabel U, Ando M, Baughman R, Cordier JF, du Bois $\mathrm{R}$, et al. ATS/ERS/WASOG statement on sarcoidosis. American Thoracic Society/European Respiratory Society/World Association of Sarcoidosis and other Granulomatous Disorders. Sarcoidosis Vasc Diffuse Lung Dis. (1999) 16:149-73.

32. Nioche C, Orlhac F, Boughdad S, Reuze S, Goya-Outi J, Robert C, et al. LIFEx: a freeware for radiomic feature calculation in multimodality imaging to accelerate advances in the characterization of tumor heterogeneity. Cancer Res. (2018) 78:4786-9. doi: 10.1158/0008-5472.CAN-18-0125

33. He JP, Hao Y, Li M, Wang J, Guo FJ. Tumor-to-background ratio to predict response to chemotherapy of osteosarcoma better than standard uptake values. Orthop Surg. (2014) 6:145-53. doi: 10.1111/os.12102

34. Lee SJ, Choi YY, Kim C, Chung MS. Correlations between tumor to background ratio on breast-specific gamma imaging and prognostic factors in breast cancer. J Korean Med Sci. (2017) 32:1031-7. doi: 10.3346/jkms.2017.32.6.1031

35. Barrington SF, Kluge R. FDG PET for therapy monitoring in Hodgkin and non-Hodgkin lymphomas. Eur J Nucl Med Mol Imaging. (2017) 44(Suppl. 1):97-110. doi: 10.1007/s00259-017-3690-8

36. Sunderland JJ, Christian PE. Quantitative PET/CT scanner performance characterization based upon the society of nuclear medicine and molecular imaging clinical trials network oncology clinical simulator phantom. J Nucl Med. (2015) 56:145-52. doi: 10.2967/jnumed.114.148056

37. Meignan M, Cottereau AS, Versari A, Chartier L, Dupuis J, Boussetta S, et al. Baseline metabolic tumor volume predicts outcome in high-tumor-burden follicular lymphoma: a pooled analysis of three multicenter studies. J Clin Oncol. (2016) 34:3618-26. doi: 10.1200/JCO.2016.66.9440

38. Erdi YE, Mawlawi O, Larson SM, Imbriaco M, Yeung H, Finn R, et al. Segmentation of lung lesion volume by adaptive positron emission tomography image thresholding. Cancer. (1997) 80(12 Suppl):2505-9. doi: 10. 1002/(SICI)1097-0142(19971215)80:12+<2505::AID-CNCR24>3.0.CO;2-F

39. Erdi YE, Wessels BW, Loew MH, Erdi AK. Threshold estimation in single photon emission computed tomography and planar imaging for clinical radioimmunotherapy. Cancer Res. (1995) 55(23 Suppl):5823s-6s.

40. Team RC, R: A Language and Environment for Statistical Computing. Vienna: R Foundation for Statistical Computing (2018).

41. Foss A, Markatou M, Ray B, Heching A. A semiparametric method for clustering mixed data. Machine Learning. (2016) 105:419-58. doi: 10.1007/s10994-016-5575-7

42. Foss A, Markatou M. kamila: clustering mixed-type data in $\mathrm{R}$ and hadoop. $J$ Statist Software. (2018) 83:1-45. doi: 10.18637/jss.v083.i13

43. Hennig C. fpc: Flexible Procedures for Clustering (2020).

44. Robin X, Turck N, Hainard A, Tiberti N, Lisacek F, Sanchez JC, et al. pROC: an open-source package for $\mathrm{R}$ and $\mathrm{S}+$ to analyze and compare ROC curves. BMC Bioinformatics. (2011) 12:77. doi: 10.1186/1471-2105-12-77

45. Zidar DA, Al-Kindi SG, Liu Y, Krieger NI, Perzynski AT, Osnard $\mathrm{M}$, et al. Association of lymphopenia with risk of mortality among adults in the US general population. JAMA Netw Open. (2019) 2:e1916526. doi: 10.1001/jamanetworkopen.2019.16526

46. Berezné A, Bono W, Guillevin L, Mouthon L. [Diagnosis of lymphocytopenia]. Presse Med. (2006) 35:895902. doi: 10.1016/S0755-4982(06)74709-1

47. Valiathan R, Deeb K, Diamante M, Ashman M, Sachdeva N, Asthana D. Reference ranges of lymphocyte subsets in healthy adults and adolescents with special mention of $\mathrm{T}$ cell maturation subsets in adults of South Florida. Immunobiology. (2014) 219:487-96. doi: 10.1016/j.imbio.2014.02.010

48. McQuillan GM, Kruszon-Moran D. HIV infection in the United States household population aged 18-49 years: results from 1999-2006. NCHS Data Brief. (2008) 1-8. doi: 10.1037/e407182008-001

49. Celada LJ, Drake WP. Targeting CD4 $(+) \mathrm{T}$ cells for the treatment of sarcoidosis: a promising strategy? Immunotherapy. (2015) 7:57-66. doi: 10.2217/imt.14.103

50. Crouser ED, Lozanski G, Fox CC, Hauswirth DW, Raveendran R, Julian MW. The CD4+ lymphopenic sarcoidosis phenotype is highly responsive to anti-tumor necrosis factor-\{alpha\} therapy. Chest. (2010) 137:14325. doi: 10.1378/chest.09-2576

51. Kamphuis LS, van Zelm MC, Lam KH, Rimmelzwaan GF, Baarsma GS, Dik WA, et al. Perigranuloma localization and abnormal maturation of B cells: emerging key players in sarcoidosis? Am J Respir Crit Care Med. (2013) 187:406-16. doi: 10.1164/rccm.201206-1024OC

52. Tøndell A, Rø AD, Børset M, Moen T, Sue-Chu M. Activated CD8+ $\mathrm{T}$ cells and natural killer $\mathrm{T}$ cells in bronchoalveolar lavage fluid in hypersensitivity pneumonitis and sarcoidosis. Sarcoidosis Vasc Diffuse Lung Dis. (2015) 31:316-24.

53. Snyder-Cappione JE, Nixon DF, Chi JC, Nguyen ML, Kirby CK, Milush JM, et al. Invariant natural killer T (iNKT) cell exhaustion in sarcoidosis. Eur J Immunol. (2013) 43:2194-205. doi: 10.1002/eji.201243185

54. Crouser ED. Role of imbalance between Th17 and regulatory T-cells in sarcoidosis. Curr Opin Pulm Med. (2018) 24:5216. doi: 10.1097/MCP.0000000000000498

Conflict of Interest: The authors declare that the research was conducted in the absence of any commercial or financial relationships that could be construed as a potential conflict of interest.

Copyright (C) 2021 Vagts, Ascoli, Fraidenburg, Baughman, Huang, Edafetanure-Ibeh, Ahmed, Levin, Lu, Perkins, Finn and Sweiss. This is an open-access article distributed under the terms of the Creative Commons Attribution License (CC BY). The use, distribution or reproduction in other forums is permitted, provided the original author(s) and the copyright owner(s) are credited and that the original publication in this journal is cited, in accordance with accepted academic practice. No use, distribution or reproduction is permitted which does not comply with these terms. 